\title{
Experience with the application of the spectral analysis of surface waves method on a falling weight deflectometer time history
}

\author{
Jozef Komacka $^{1, *}$ \\ ${ }^{1}$ University of Žilina, Faculty of Civil Engineering, Univerzitná 8215/1, 01026 Žilina, Slovakia
}

\begin{abstract}
Falling Weight Deflectometers are commonly used in pavement diagnostics and the outputs of measurements are a basis for pavement performance evaluation. Usually, a surface modulus of a pavement is determined or the modulus of a pavement layer is back-calculated using maximum values of a load force and measured deflections. A Falling Weight Deflectometer time history contains data describing a total response of a pavement to a load in time domain. The theory and principles of the Spectral Analysis of Surface Waves method were applied to these measurement outputs to determine Rayleigh wave velocity and a compression-tension modulus of a pavement. The problems with the determination of travel time of a wave were identified. The calculated compression-tension modulus differed noticeably depending on a used distance between receivers. Analysis showed the depth of the propagation of a wave or the bulk density of an investigated area is probably not a reason of the difference in the values of the compression-tension modulus calculated for the various distances of receivers.
\end{abstract}

\section{Introduction}

Falling Weight Deflectometrs (FWD) are commonly used in pavement bearing capacity diagnostic and the outputs of measurements are a basis for performance evaluation of a pavement structure. The outputs of diagnostics by a FWD contain a lot of data regarding the change of a load force and deflections values in time domain. This time history describes total pavement response to a load. Common practice in pavement performance evaluation is to use only the maximum values of a load force and deflections measured at various spacing. A surface modulus is calculated from the peak value of a load and deflection at the axis of a load plate using the linear elastic half space theory. It characterizes total stiffness of a pavement and its subgrade without possibility to determine which part of the system is weak when a pavement has low performance. This disadvantage can be eliminated via back-calculation of modulus of pavement layers and subgrade based on the multilayered linear elastic system theory or finite element methods. Both mentioned approaches use the static interpretation of a load although it is dynamic in reality. Modeling of a pavement response to a dynamic load in a pavement model loaded by an impulse with

* Corresponding author: komacka@,fstav.utc.sk 
a shape comparable to a FWD load impulse [1] indicated problems with the boundary conditions of the calculation model and longtime of calculations.

The application of the Spectral Analysis of Surface Waves (SASW) method is another possibility to respect dynamic nature of the FWD load. The SASW method consists of generation, measurement and processing of dispersive elastic waves in a layered system. Dispersion of one kind of surface waves, named Rayleigh's waves, is used to assess the stiffness of an investigated system. The application of the SASW method consists of field measurements of surface wave velocity at numerous wavelengths. The typical configuration to perform field measurements using the SASW method consists of a source, one [2], two or more vertical acceleration transducers located on a surface at the equidistance from the source and a data acquisition system. A vertical load is applied to generate surface waves and the propagation of these waves along a surface is recorded by receivers. A pavement bearing capacity diagnostics procedure by a FWD is basically the same as investigation by the SASW method. A vertical load is generated by a falling mass and pavement response is recorded by sensors spaced in different distances from a load axis. Number of the sensors obviously varies from 5 to 8 and they are positioned at various distances up to $2.1 \mathrm{~m}$ from the axis of a load. Due to similarity of the principles of both methods the theory used for evaluation of SASW measurements was applied to FWD outputs.

\section{Theoretical backgrounds}

Rayleigh's waves are two dimensional surface waves generated by a vertical impulse load. They consist of longitudinal and transversal oscillations that propagate in a plane that is predetermined by the direction of a wave. The velocity of Rayleigh's wave can be determined using the velocity of a shear wave, the correlation or spectral analysis methods or direct determination of Rayleigh's wave velocity. The last one is very convenient approach from practical point of view. The velocity of Rayleigh's wave $c_{R}$ is determined from the formula

$$
c_{R}=L / t
$$

where $L=$ the distance of the receivers used for determination of a wave velocity; and $t=$ travel time of a wave between the receivers.

Since the distance of the receivers is always known, only the response of the receivers in time domain has to be recorded. Moreover, the distance between various receivers can be used to determine the wave velocity.

The known velocity of the Rayleigh's wave is a base to calculate a shear modulus $G$ using the formula

$$
G=\rho \cdot\left(c_{R} / r_{v}\right)^{2}
$$

where $\rho=$ the bulk density of investigated area; and $r_{v}=$ the velocity ratio of Rayleigh `s and shear wave.

Subsequently, compression-tension modulus $E$ can be calculated by

$$
E=2 \cdot G(1+\mu)
$$

where $\mu=$ Poisson`s ratio of investigated area.

As for surface waves (i.e. also for Rayleigh's waves) it is important that energy of a wave reduces along a depth. The depth of propagation of a Rayleigh's wave depends on its wavelength $\lambda_{R}$ that is a function of the velocity $c_{R}$ of the Rayleigh's wave and the frequency of a load impulse $f_{R}$ 


$$
\lambda_{R}=c_{R} / f_{R}
$$

Waves with short wavelength propagate near ground surface whereas longer wavelengths travel deeper. Experience indicates the depth of the propagation of Rayleigh's waves is approximately equal to its wavelength.

\section{Experimental}

The compression-tension modulus of a pavement structure was determined based on the theory of the SASW method. The essential input, the velocity of the Rayleigh's wave, was calculated using the FWD time history data. There are data relating to time change of a load impulse and surface deflections at known distances from the axis of a load plate (Fig. 1).

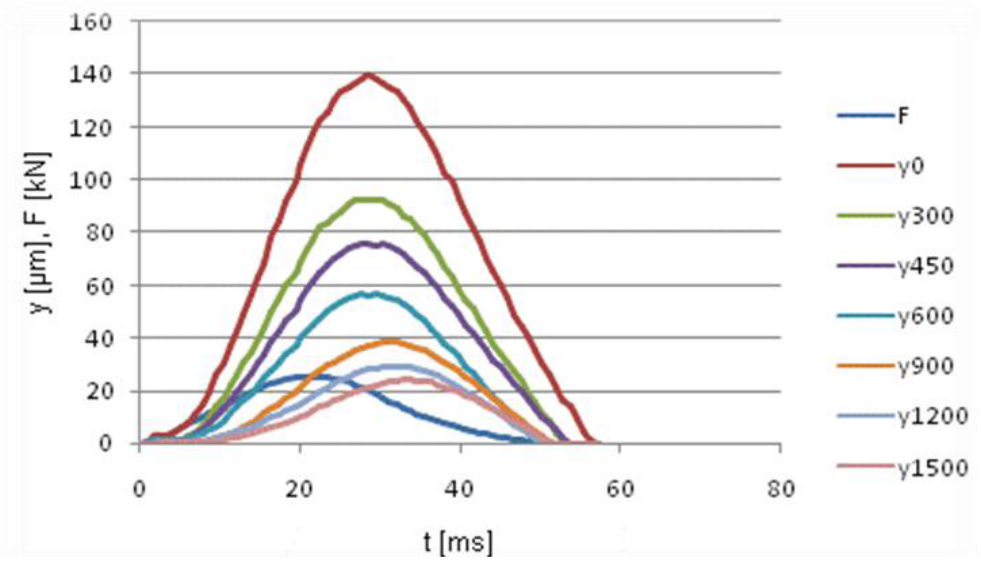

Fig. 1. Example of FWD time history.

The travel time of a Rayleigh's wave between receivers can be determined using the front of a wave or the maximum value of a surface deflection (Fig. 2). The analysis of the time history data showed the use of the maximal value of a surface deflection is a better approach because it was not possible to exactly determine the arrival time of a wave at the positions of the receivers. The probably reason was that the small changes of the deflections at beginning were not properly registered due to accuracy (sensitivity) of the FWD receivers. Next problem was identified when the maximal values of the deflections were intended to use. There were the same values at the consecutive time records. Therefore, the approximation of the course of the values close to a maximum value was performed (Fig. 3). The maximum deflection value and relating time was determined using the approximation curve. When time of the maximum values determined by the approximation was plotted against the distance of the FWD receivers it was found in some cases that time not increased with the distance as it was expected. Therefore, the regression curve was derived to determine final time of the maximum values of the deflections at the FWD receivers (Fig. 4). These values were used to calculate the velocity of Rayleigh's wave using known spacing of the FWD receivers. 


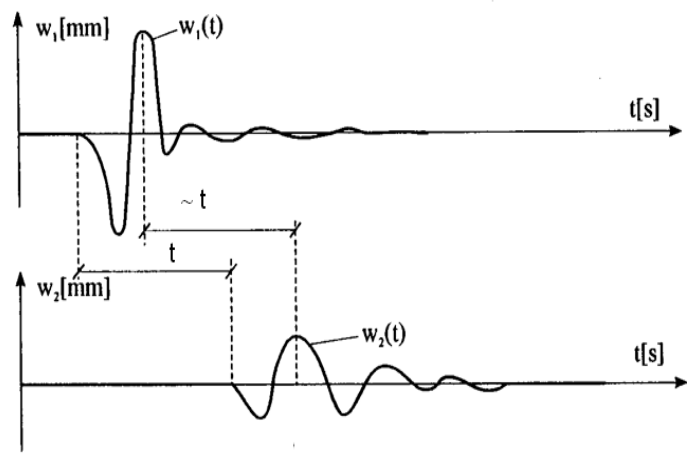

Fig. 2. Determination of travel time of wave.

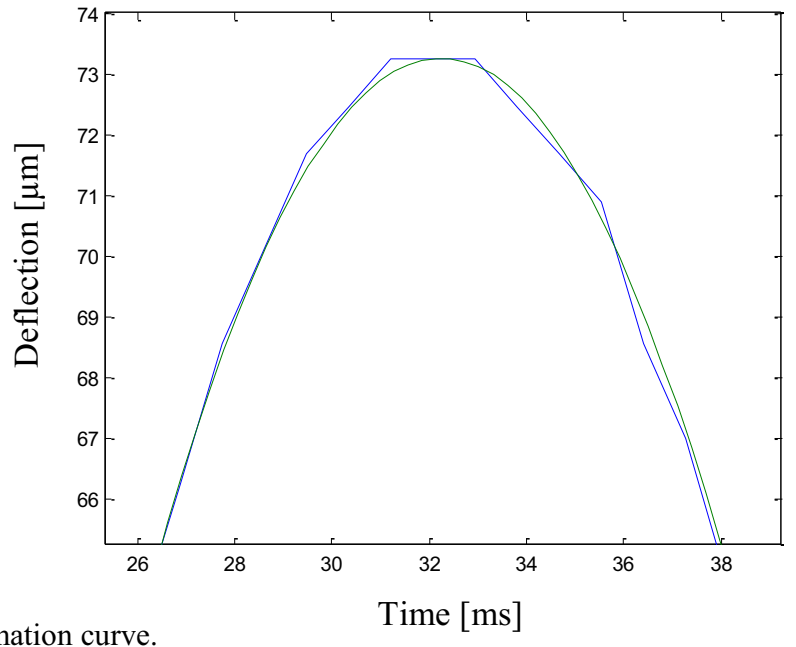

Fig. 3. The approximation curve.

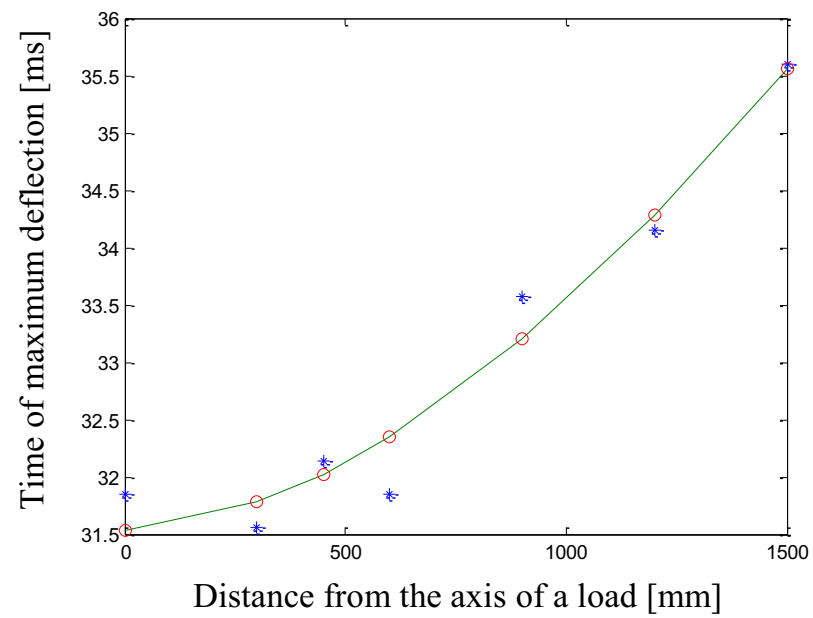

Fig. 4. Time of the maximum deflections at the FWD receivers. 
The shear modulus and afterwards compression-tension modulus was calculated for the distance between the receiver positioned at the axis of the FWD load plate and others receivers that were placed in a line on a beam in the distance of $300 \mathrm{~mm}, 450 \mathrm{~mm}, 600 \mathrm{~mm}$, $900 \mathrm{~mm}, 1200 \mathrm{~mm}$ and $1500 \mathrm{~mm}$ from the axis of the FWD load plate. The compressiontension modulus calculated for six different distances of the FWD receivers from the axis of a load is plotted in Fig. 5.

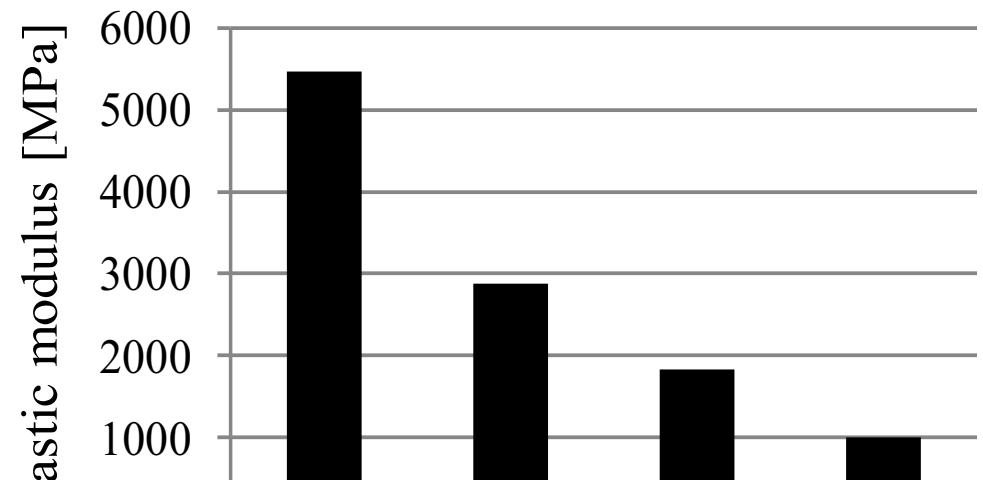

Fig. 5. Compression-tension modulus calculated for the different distances of a receiver to the axis of a load.

It is evident the compression-tension modulus values are strong different and decrease when the distance increases. It means the calculated velocity of a Rayleigh's wave was different when the various spacing of the receivers was used. Assuming the problem could be connected with the use of the receiver in the axis of a load the receiver at the distance of $300 \mathrm{~mm}$ was taken into account as a base and new distances to the more distant receivers were used for the calculation of Rayleigh's wave velocity and both modulus. Nevertheless, the results were basically the same (Fig. 6). This finding was also verified for next numerous distances calculated from others possible combinations of the receivers.

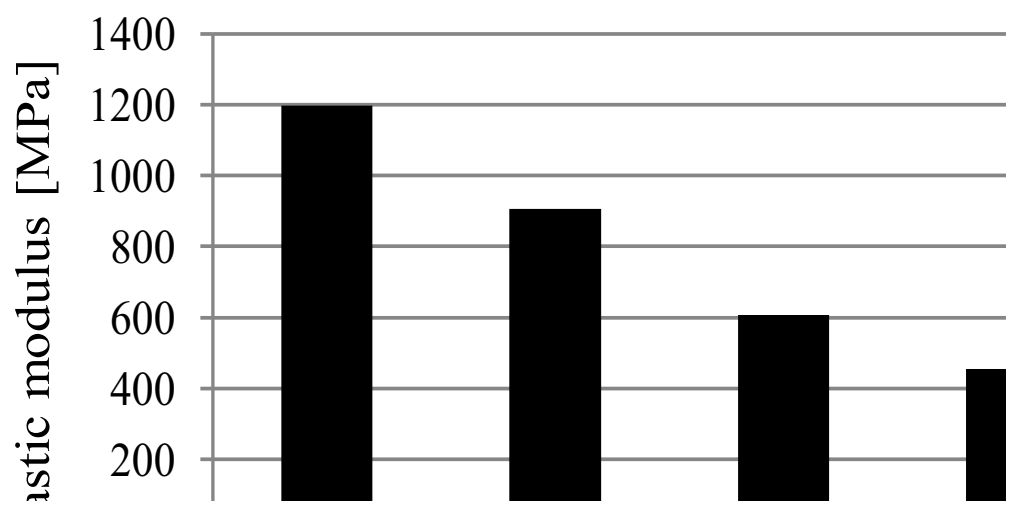

Fig. 6. Compression-tension modulus calculated for the various distance of a receiver to the receiver at $300 \mathrm{~mm}$ from the axis of a load.

Keeping in mind the frequency of a load impulse can influence the wavelength of Rayleigh's wave and the depth of its propagation the load impulse of used FWD was analysed. Data in time domain (see Fig. 1, the blue line labelled as "F") was transferred into frequency domain using the discrete Fourier transformation (DFT). The result confirmed an 
expectation and only one dominant frequency was identified in the FWD load impulse. When the same value of a frequency is used in the formula (4), the different velocity of a wave leads to a different wavelength. As a wavelength relate to the depth of the propagation of a wave it could be assumed the differences in the depth of the propagation of a wave is a cause of the difference in the values of compression-tension modulus. However, the wavelength (and subsequently the depth of the propagation of a wave) is directly proportional to the velocity of a wave. It means a higher velocity leads to a higher wavelength and deeper propagation. Based on this, the differences in the values of compression-tension modulus should relate to material differences in great depth (more than $15 \mathrm{~m}$ in this case). As it does not seem real attention was drawn to the calculation of a shear modulus according to the formula (2).

In addition to the speed of a wave the bulk density of area is another input parameter. The influence of a bulk density to the velocity of a wave could be found in [3]. In this case the same value of the bulk density was used for all distances of the receivers. It resulted to the different values of shear modulus due to the dissimilar velocities of the wave. If the values of the shear modulus should be the same for any distance of the receivers (assuming the homogeneity of investigated area) the values of the bulk density has to be different. This assumes a wave propagates to different depths and a bulk density changes along the depth. It is only possible in a layered system with the different bulk density of layers. This is a real situation in the case of asphalt pavements. Therefore, the bulk densities were calculated to have the same value of a shear modulus regardless the distance of the receivers. The values of the shear modulus were chosen from the range of the values calculated in the previous evaluation. A part of outputs is shown in Table1.

Table 1. Calculated bulk density for variable values of constituents.

\begin{tabular}{|c|c|c|c|c|c|c|}
\hline & \multicolumn{6}{|c|}{ Distance of receivers in mm } \\
\cline { 2 - 7 } & 300 & 450 & 600 & 900 & 1200 & 1500 \\
\hline Shear modulus & \multicolumn{7}{|c|}{$2025 \mathrm{MPa}$} \\
\hline $\begin{array}{c}\text { Wave velocity } \\
\text { in m/s }\end{array}$ & 985 & 714 & 570 & 421 & 345 & 299 \\
\hline $\begin{array}{c}\text { Bulk density in } \\
\text { g/cm }\end{array}$ & 2.087 & 3.972 & 6.233 & 11.425 & 17.013 & 22.651 \\
\hline \begin{tabular}{c} 
Shear modulus \\
\hline $\begin{array}{c}\text { Wave velocity } \\
\text { in m/s }\end{array}$
\end{tabular} & 985 & 714 & 570 & 421 & 345 & 299 \\
\hline $\begin{array}{c}\text { Bulk density in } \\
\text { g/cm }\end{array}$ & 0.192 & 0.365 & 0.572 & 1.049 & 1.563 & 2.081 \\
\hline
\end{tabular}

The calculated bulk densities are very different and mostly unrealistic (very high or very low) when compared to the real bulk density (approx. $2.084 \mathrm{~g} \cdot \mathrm{cm}^{-3}$ ). It cannot be concluded the variability of the shear modulus calculated for the various distances of the receiver relate to the differences in the bulk density of investigated area.

\section{Conclusions}

The application of the SASW method on the FWD time history data indicated some problems. It was not possible to exactly determine the arrival time of a wave when the front of a wave was chosen. The better solution was to use the maximum value of a pavement 
surface deflection. Nevertheless, an approximation was necessary to determine final time of the maximum values of the deflections at the receivers.

The compression-tension modulus calculated from the various distances of the receivers differed noticeably. The higher values were determined for the shorter distances of the receivers. The analysis of the load impulse in frequency domain proved the existence only one dominant frequency. Therefore, the possible influence of the depth of the propagation of a wave was assessed. Taking into account the calculated wavelength the depth of the propagation of a wave was estimated. It was found the material differences in the great depth should be a reason of difference in the values of compression-tension modulus.

As this is probably not real the influence of the bulk density of investigated area was analysed. It was concluded the calculated values are very different and mostly unrealistic and therefore they could not be the cause of the variability of the shear modulus calculated for the various distances of receivers.

The author is grateful for the financial support from the research project VEGA 1/005/16 Modelling of selected dynamic problems in time and frequency domain

\section{References}

1. N. Rapanová, J. Kortiš, Transport and telecommunication 14 (3), 230-236 (2013)

2. N. Ryden et al, J Geotech Geoenviron 130 (6), 636-645 (2004)

3. J. Komačka, N. Rapanová, Road horizon (Silniční obzor) 73 (7-8), 205-209 (2012) 